\title{
Reduction of muscle fatigue by catchlike-inducing intermittent electrical stimulation in rat skeletal muscle
}

\author{
Yoichi Shimada ${ }^{1}$, Hiroki Ito ${ }^{2}$, Toshiki Matsunaga ${ }^{1}$, Akiko Misawa ${ }^{2}$, Masahito Kawatani ${ }^{3}$ and Eiji Itol $^{2}$ \\ ${ }^{1}$ Rehabilitation Division, ${ }^{2}$ Department of Orthopaedic Science, Section of Neuro and Locomotor Science, and ${ }^{3}$ Department of Physiol- \\ ogy, Akita University School of Medicine, Akita 010-8543, Japan
}

(Received 23 May 2006; and accepted 20 June 2006)

\begin{abstract}
Catchlike property is the force enhancement produced when a brief, high-frequency burst of pulses is added to a constant low-frequency stimulation. In functional electrical stimulation, constant low-frequency stimulation of approximately $20 \mathrm{~Hz}$ has primarily been used to reduce muscle fatigue. The purpose of this study was to investigate the effects of catchlike-inducing intermittent stimulation on muscle fatigue in relation to continuous intermittent low-frequency stimulation. Twenty-two adult male Wistar ST rats were randomly assigned into the constant frequency stimulation (CFS) group or the catchlike-inducing stimulation (CIS) group. In the CFS group, constant low-frequency stimulation of $20 \mathrm{~Hz}$ was applied intermittently (4 seconds "ON"/15 seconds "OFF"). In the CIS group, a single electrical burst of $100 \mathrm{~Hz}$ was applied at the start of the every 4-second period of stimulation. The muscle fatigue test lasted for $16 \mathrm{~min}$ and isometric muscle force, muscle fatigue, and muscular workload were evaluated. CIS significantly increased the maximum muscular force (under fatigued condition) and workload, and significantly decreased muscle fatigue $(p<0.05)$. The results of this study suggest that catchlike-inducing intermittent electrical stimulation is useful in the clinical administration of functional electrical stimulation.
\end{abstract}

Functional electrical stimulation (FES) is the technology of restoring voluntary control of movement in patients who have central nervous system (CNS) dysfunction and for whom no normal recovery is expected (19). Muscles paralyzed by CNS dysfunction can be electrically activated through their surviving motor nerve supply, thus providing the possibility of improving impaired motor function by FES (17). In the first trial of FES, Liberson and colleagues reported that hemiplegic patients improved their walking when the peroneal nerve was stimulated during the swing phase of the gait (14). Today, FES makes it possible for patients with paraplegia

Address correspondence to: Yoichi Shimada, MD, Rehabilitation Division, Akita University Hospital, 1-1-1 Hondo, Akita 010-8543, Japan

Tel: +81-18-884-6147, Fax: +81-18-884-6147

E-mail: yshimada@med.akita-u.ac.jp caused by spinal cord injury to regain the abilities of standing and walking $(1,9,13,15,21)$.

When FES restored the functions of the lower extremities, muscle fatigue usually occurred much earlier than under normal physiological conditions. In restoration of standing and walking, muscle fatigue leads to knee buckling, thus hindering restorative efforts $(1,13,21)$. In the present clinical FES systems, smooth muscular contraction and functional restoration are primarily achieved through continuous stimulation. At the same time, it is necessary to apply stimulation at frequencies that are less likely to cause muscle fatigue, so low-frequency stimulation (typically at less than $50 \mathrm{~Hz}$ ) is used $(13,15$, 21). Currently, a major factor limiting the widespread clinical application of FES is the rapid muscle fatigue that accompanies its use (3). Recent advances in medical engineering technology have made it possible to perform more efficient intermit- 
tent FES incorporating closed-loop control $(9,22)$. The strength of contraction and the rate of fatigue are both directly related to stimulation frequency (3). In the closed-loop FES system, the optimal intermittent stimulation frequency for restoring leg function has not been conclusively determined.

The "catchlike" property of skeletal muscle is the tension enhancement seen when an initial brief highfrequency burst of pulses at the onset of a constant frequency train is used to activate a whole muscle or single motor units (5). Burke and colleagues applied a single electrical stimulation of $100 \mathrm{~Hz}$ at the start of low-frequency stimulation of excised feline muscles, and reported increased and sustained muscle force for the first time (8). This phenomenon was later confirmed by various researchers and is now referred to as the "catchlike" property of muscle $(2,3,23,24)$. Bevan and colleagues reported that when the first two stimuli were delivered at an instantaneous frequency of $100 \mathrm{~Hz}$, followed by a stimulus train at $20 \mathrm{~Hz}$, catchlike-inducing stimulation produced higher forces and better force maintenance compared with constant frequency stimulation in the fast motor units of cats (2). From these studies, there is a possibility that the catchlike-inducing intermittent stimulation of whole skeletal muscle can reduce muscle fatigue rather than constant frequency stimulation.

The purpose of this study was to investigate the effects of catchlike-inducing intermittent stimulation on muscle fatigue in relation to continuous stimulation.

\section{MATERIALS AND METHODS}

Surgical procedure. The medial gastrocnemius muscles of adult male Wistar ST rats were used in this experiment. Each rat was deeply anaesthetized with an intraperitoneal injection of pentobarbital sodium $(30 \mathrm{mg} / \mathrm{kg})$. The animal was then mounted in a rigid frame that securely immobilized the test (right) leg and pelvis. The skin over the posterior leg and popliteal space was reflected and the sciatic nerve and the medial gastrocnemius were exposed. A commercially available bipolar cuff electrode (inter-electrode distance : $5 \mathrm{~mm}$; MD Giken, Tokyo, Japan) was attached to the sciatic nerve at the center of the femur. The medial gastrocnemius was severed at the tendon near the attachment site to the calcaneus. A transducer (ORIENTEC Co., Tokyo, Japan) was attached to the end of the medial gastrocnemius and fixed with a load of approximately $1 \mathrm{~N}$ next to the stump (11). Signals transmitted from the force trans- ducer during isometric muscular contraction were recorded on a force-time curve using a paper recorder (Nihon Kohden Co., Tokyo, Japan). To prevent the muscles from drying during the study, mineral oil was used to cover the exposed area (4). The study was conducted at a constant temperature of $25-27^{\circ} \mathrm{C}$ and the rectal temperature of the rats was maintained at $35-36^{\circ} \mathrm{C}$. At the end of the study, the rats were euthanized by carbon monoxide inhalation. The protocols for animal experimentation described in this paper were previously approved by the Animal Research Committee, Akita University; all subsequent animal experiments adhered to the "Guidelines for Animal Experimentation" of the university.

Experimental protocol. Twenty-two adult male Wistar ST rats with an average body weight of $287 \pm$ $19.8 \mathrm{~g}$ were randomly assigned to two groups: the constant frequency stimulation (CFS) group $(n=11)$ and the catchlike-inducing stimulation (CIS) group $(\mathrm{n}=11)$. In the CFS group, 4-second constant low-frequency stimulation of $20 \mathrm{~Hz}$ applied intermittently. In the CIS group, 4-second constant low-frequency stimulation of $20 \mathrm{~Hz}$ was applied intermittently, and at the start of the every 4-second period of stimulation, a single electrical burst of $100 \mathrm{~Hz}$ was applied. In both the CFS and CIS groups, a constant monophasic rectangular pulse with a pulse width of $0.2 \mathrm{msec}$ and a constant stimulation intensity of $-4 \mathrm{~V}$ was used.

Before the muscle fatigue test, the maximum potentiation effect (constant maximum isometric muscle force) was confirmed by applying low-frequency stimulation of $20 \mathrm{~Hz}$ for 4 seconds (4-second "on" period) followed by a 15 -second "off" period; this procedure was repeated 4-5 times (4). The muscle fatigue test was then conducted as follows. In the CFS group, low-frequency stimulation of $20 \mathrm{~Hz}$ was applied for 4 seconds with a break of 15 seconds before the next stimulation. This procedure was repeated 50 times to record the isometric muscle force for a 16-minute period. Similarly, in the CIS group, the isometric muscle force was recorded to ascertain the catchlike-inducing effects.

Data management and analysis. The maximum isometric muscle force $\left(\mathrm{F}_{\mathrm{PEAK}}\right.$, unit: $\left.\mathrm{N}\right)$, the muscle force at the fourth second $\left(\mathrm{F}_{4 \mathrm{sec}}\right.$, unit: $\left.\mathrm{N}\right)$ (Fig. 1), and the integral muscle force (force-time integral: FTI, unit: NS) (Fig. 2) were determined for each 4-second muscle force-time curve during the muscle fatigue test (6). The following three FTI parameters 


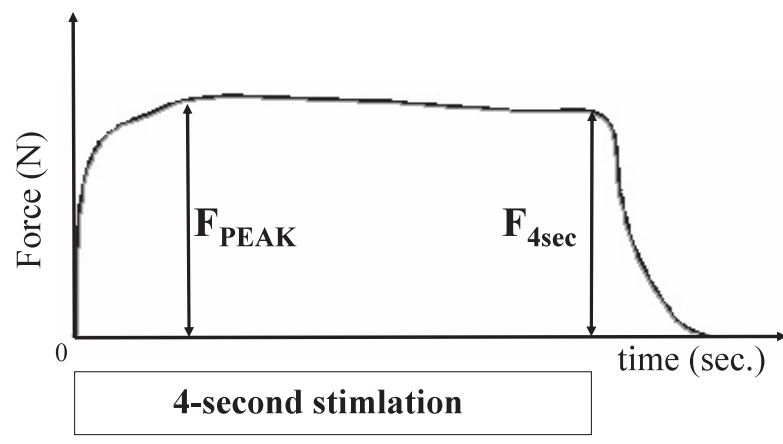

Fig. 1 The force time curve during 4-second stimulation. Maximum isometric muscle force $\left(F_{\mathrm{PEAK}}\right)$ and muscle force at the fourth second just before start of the "off" period $\left(F_{4 \mathrm{sec}}\right)$ were determined as shown.

were calculated: FTI for the entire 4-second stimulation period $\left(\mathrm{FTI}_{0-4}\right.$, Fig. 2a), FTI from start of stimulation to one second after start of stimulation (FTI ${ }_{0-1}$, Fig. 2A), and FTI from one second after start of stimulation to end of stimulation $\left(\mathrm{FTI}_{1-4}\right.$, Fig. 2B). $\mathrm{FTI}_{0-4}$ represents the muscular workload for the entire 4-second period, $\mathrm{FTI}_{0-1}$ represents the start-up muscular workload soon after stimulation, and $\mathrm{FTI}_{1-4}$ represents the late-stage muscular workload. These FTI parameters were calculated using image software analysis (Mac Scope version 2.5; Mitani Corporation, Chiba, Japan).

Muscle fatigue was defined as attenuation in $\mathrm{F}_{\text {PEAK }}$ (maximum muscle force) at the $\mathrm{n}^{\text {th }} 4$-second stimulation $\left[\mathrm{F}_{\text {PEAK }}(\mathrm{n})\right]$ in relation to the $\mathrm{F}_{\mathrm{PEAK}}$ at the initial 4-second stimulation in the muscle fatigue test $\left[F_{\text {PEAK }}(i)\right]$. To ascertain the time course of muscle fatigue, the percentage of initial $\mathrm{F}_{\text {PEAK }}(\mathrm{n})$ was calculated using the formula

$$
\begin{aligned}
& \text { Percentage of initial } \mathrm{F}_{\text {PEAK }}(\mathrm{n})= \\
& \qquad\left[\mathrm{F}_{\mathrm{PEAK}}(\mathrm{n}) / \mathrm{F}_{\text {PEAK }}(\mathrm{i})\right] \times 100
\end{aligned}
$$

To assess the time course of muscle fatigue at the end of each 4-second stimulation, attenuation in $\mathrm{F}_{4 \mathrm{sec}}$ at the $\mathrm{n}^{\text {th }}$ stimulation $\left[\mathrm{F}_{4 \mathrm{sec}}(\mathrm{n})\right]$ in relation to that at the initial 4-second stimulation in the muscle fatigue test $\left[\mathrm{F}_{4 \mathrm{sec}}(\mathrm{i})\right]$ was calculated using the formula

$$
\begin{aligned}
& \text { Percentage of initial } \mathrm{F}_{4 \mathrm{sec}}(\mathrm{n})= \\
& \qquad\left[\mathrm{F}_{4 \mathrm{sec}}(\mathrm{n}) / \mathrm{F}_{4 \mathrm{sec}}(\mathrm{i})\right] \times 100
\end{aligned}
$$

The percentage of FTI at the $\mathrm{n}^{\text {th }}$ stimulation [FTI(n)] to the FTI at the initial 4-second stimulation in the muscle fatigue test [FTI(i)] was calculated using the formula

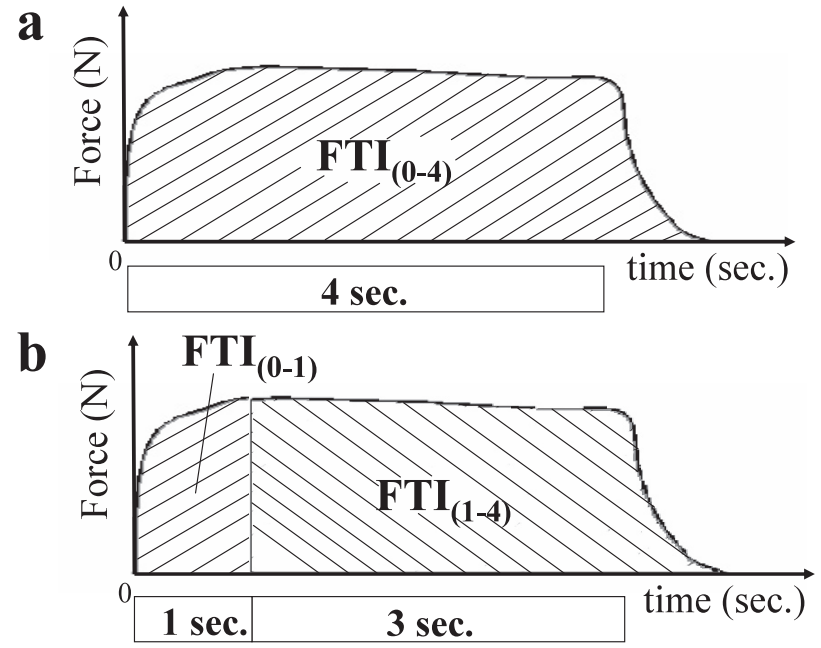

Fig. 2 Calculation of the force-time integral (FTI) from the force-time curves. a) $\mathrm{FTI}_{(0-4)}$ indicates $\mathrm{FTI}$ for the entire 4-second stimulation period. b) $\mathrm{FTI}_{(0-1)}$ and $\mathrm{FTI}_{(1-4)}$ measurement methods. $\mathrm{FTI}_{(0-1)}$ indicates FTI from start of stimulation to one second after start of stimulation and $\mathrm{FTI}_{(1-4)}$ indicates FTI from one second after start of stimulation to end of stimulation.

Percentage of initial FTI (n) =

$[\mathrm{FTI}(\mathrm{n}) / \mathrm{FTI}(\mathrm{i})] \times 100$

The time courses of the three parameters $F(0-4)$, $\mathrm{F}(0-1)$, and $\mathrm{F}(1-4)$ were determined for every ten stimulations.

Statistical analysis. Significant differences in the maximum muscle force over the 4-second stimulation period $\left(\mathrm{F}_{\text {PEAK }}\right)$ and the muscle force at the fourth second $\left(\mathrm{F}_{4 \mathrm{sec}}\right)$ between CFS and CIS groups at the start and end of the muscle fatigue test were evaluated using a $t$-test with a significance level of $5 \%$.

Repeated measure ANOVA (Stat-View version 5; Cary, NC, USA) was used to statistically analyze differences in the percentage of initial FPEAK (n), percentage of initial $\mathrm{F}_{4 \mathrm{sec}}(\mathrm{n})$, and percentage of initial FTI between CFS and CIS groups at a significance level of $5 \%$. Numerical values were expressed as mean \pm standard deviation.

\section{RESULTS}

Muscle force measurements

At the start of the muscle fatigue test, the $\mathrm{F}_{\text {PEAK }}$ was $5.75 \pm 0.76 \mathrm{~N}$ (mean $\pm \mathrm{SD}$ ) for the CFS group and $5.68 \pm 0.41 \mathrm{~N}$ for the CIS group; this difference was not statistically significant ( $t$-test, $p=0.7966)$. At the 
end of the muscle fatigue test, the $\mathrm{F}_{\mathrm{PEAK}}$ value was $2.61 \pm 0.48 \mathrm{~N}$ for the CFS group and $3.29 \pm 0.48 \mathrm{~N}$ for the CIS group; this difference was statistically significant ( $t$-test, $p=0.0050)$. No significant differences were found between the CFS and CIS groups for the $\mathrm{F}_{\text {4sec }}$ at the start of the muscle fatigue test (5.50 $\pm 0.76 \mathrm{~N}$ for the CFS group and $5.58 \pm 0.37 \mathrm{~N}$ for the CIS group; $t$-test, $p=0.7832$ ) or at the end of the muscle fatigue test $(2.12 \pm 0.31 \mathrm{~N}$ for the CFS group and $2.39 \pm 0.30 \mathrm{~N}$ for the CIS group; $t$-test, $p=0.0639$ ) (Table 1). CIS significantly increased the maximum muscular force under fatigued condition. Force-time curves for one case from each of the CFS and CIS groups at the start and end of the muscle fatigue test are shown in Fig. 3.

\section{Time course of muscle fatigue}

With time, the percentage of initial $\mathrm{F}_{\mathrm{PEAK}}$ decreased for both groups and the percentage of initial $\mathrm{F}_{\text {PEAK }}$ became significantly greater for the CIS group (repeated measure ANOVA, $p<0.0001$ ) (Fig. 4), indicating that the level of muscle fatigue for the CIS group was significantly reduced. At the end of the muscle fatigue test, the percentage of initial $F_{\text {PEAK }}$ was $45.4 \pm 5.2 \%$ for the CFS group and $57.9 \pm 5.6 \%$ for the CIS group. There were no significant differ-

Table 1 Muscle force at the start and end of the muscle fatigue test

\begin{tabular}{|c|c|c|c|c|}
\hline & \multicolumn{2}{|c|}{$\mathrm{F}_{\text {PEAK }}$} & \multicolumn{2}{|c|}{$\mathrm{F}_{4 \mathrm{sec}}$} \\
\hline & CFS Group (N) & CIS Group (N) & CFS Group (N) & CIS Group(N) \\
\hline \multirow[t]{2}{*}{ Start } & $5.75 \pm 0.76$ & $5.68 \pm 0.41$ & $5.50 \pm 0.76$ & $5.58 \pm 0.37$ \\
\hline & \multicolumn{2}{|c|}{ N.S. } & \multicolumn{2}{|c|}{ N.S. } \\
\hline \multirow[t]{2}{*}{ End } & $2.61 \pm 0.48$ & $\begin{array}{c}3.29 \pm 0.48 \\
\end{array}$ & $\begin{array}{c}2.12 \pm 0.31 \\
\llcorner\end{array}$ & $2.39 \pm 0.30$ \\
\hline & \multicolumn{2}{|c|}{$*$} & \multicolumn{2}{|c|}{ N.S. } \\
\hline
\end{tabular}

(Mean $\pm \mathrm{SD}$ )

N.S. : not significant

$* \mathrm{p}=0.005$
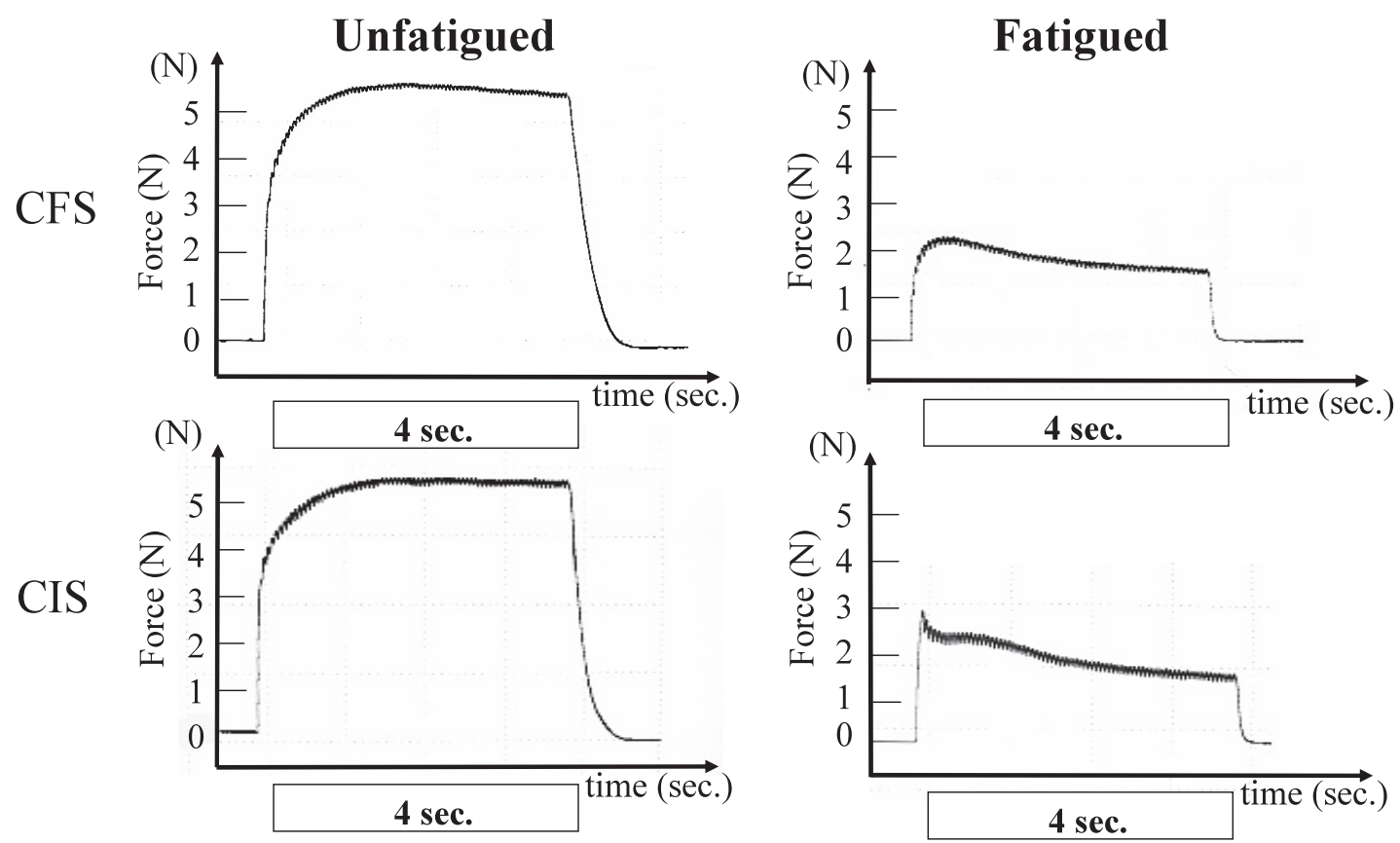

Fig. 3 Representative force-time curves at start and end of the muscle fatigue test for the CFS and CIS groups. At the start of the muscle fatigue test (unfatigued), shifts in muscle force were similar for the CFS and CIS groups. Under fatigued condition, the muscle force increased more quickly and remained higher in the CIS group, but there was no marked difference in muscle force just before the completion of the stimulation between the CFS and CIS groups. 


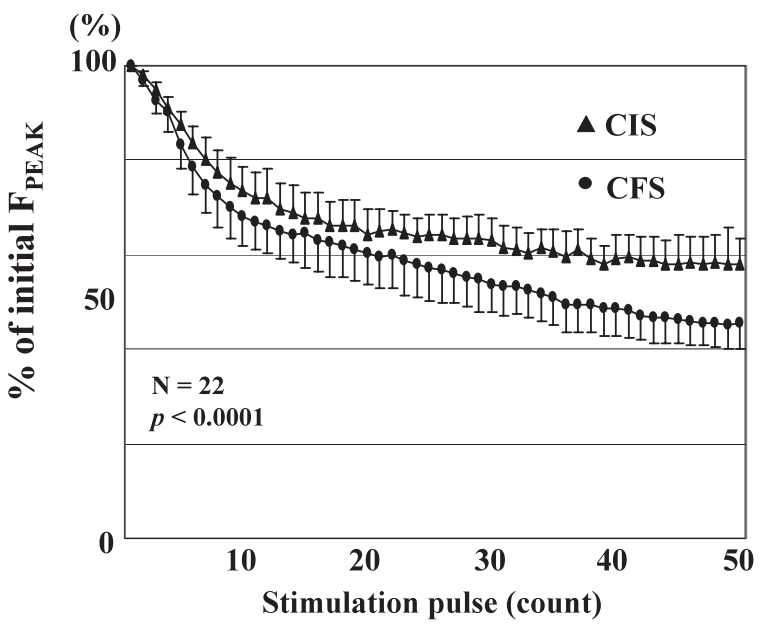

Fig. 4 Time course of $\%$ of initial $F_{\mathrm{PEAK}}$ for CFS and CIS groups during the muscle fatigue test. Differences in muscle fatigue between CFS and CIS were statistically significant (repeated measure ANOVA; $p<0.0001$ ), and muscle fatigue was greater for CFS. Symbols and bars represent mean \pm SD.

ences in the percentage of initial $\mathrm{F}_{4 \mathrm{sec}}$ between the two groups (repeated measure ANOVA, $p=0.2870$ ) (Fig. 5). At the end of the muscle fatigue test, the percentage of initial $\mathrm{F}_{4 \mathrm{sec}}$ was $38.5 \pm 3.0 \%$ for the CFS group and $42.7 \pm 4.0 \%$ for the CIS group.

Time course of muscular workload

With time, the percentage of initial $\mathrm{FTI}_{0-4}$ decreased for both groups, and that for the CIS group was significantly higher (repeated measure ANOVA, $p=0.0017$ ) (Fig. 6). At the end of the muscle fatigue test, the percentage of initial $\mathrm{FTI}_{0-4}$ was $41.6 \pm$ $4.4 \%$ for the CFS group and $46.1 \pm 6.5 \%$ for the CIS group. Also, the percentage of initial $\mathrm{FTI}_{0-1}$ increased for both groups, but the workload for the CIS group was significantly higher (repeated measure ANOVA, $p=0.0228$ ) (Fig. 7). At the end of the muscle fatigue test, the percentage of initial $\mathrm{FTI}_{0-4}$ was $49.4 \pm 7.2 \%$ for the CFS group and $56.0 \pm 8.1 \%$ for the CIS group. Furthermore, the percentage of initial $\mathrm{FTI}_{1-4}$ increased for both groups and became significantly higher for the CIS group (repeated measure ANOVA, $p=0.0122$ ) (Fig. 8). At the end of the muscle fatigue test, the percentage of initial $\mathrm{FTI}_{1-4}$ was $39.3 \pm 3.8 \%$ for the CFS group and 42.8 $\pm 6.5 \%$ for the CIS group.

\section{DISCUSSION}

In this study, the results showed that CIS significantly increased the maximum muscular force (under

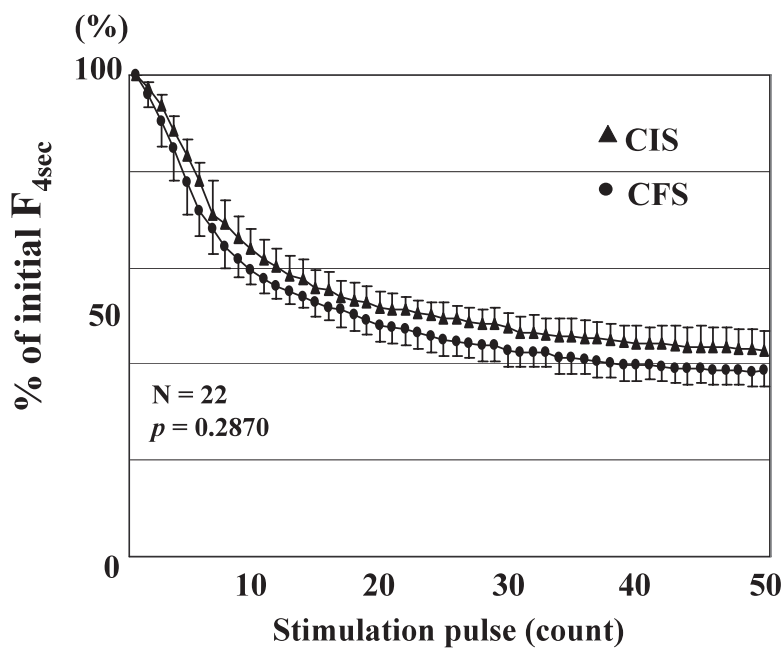

Fig. 5 Time course of $\%$ of initial $F_{4 s e c}$ for CFS and CIS during the muscle fatigue test. No statistical difference was found (repeated measure ANOVA; $p=0.2780$ ). Symbols and bars represent mean $\pm S D$.

fatigued condition) and workload, and significantly decreased muscle fatigue. According to previous reports, the effect of CIS on muscle fatigue has been confirmed for short-term stimulation of less than one second in a short cycle $(3-5,20,23)$. BinderMacleod and colleagues investigated the catch-like property of the quadriceps femoris of healthy individuals, and reported that isometric muscle force was increased by applying continuous stimulation with a duration of $300 \mathrm{msec}$ every second (3). Ratkevicius and colleagues intermittently stimulated the quadriceps femoris of healthy individuals by applying short-term $(300 \mathrm{msec})$ continuous stimulation at the subtetanic low frequency of $14.3 \mathrm{~Hz}$ every 2.5 seconds. They reported that the catch-like property increased the maximum isometric muscle force and workload, and that this effect was marked when the muscle was fatigued (20). These findings suggest that CIS could be used for controlling muscle fatigue in FES. However, previous reports only investigated short-term stimulation with short cycles and there have been no studies on CIS associated with intermittent FES using long-term electrical stimulation lasting several seconds, which is required for FES in clinical settings.

There are several clinical studies reported about the stimulation period for the restoration of standing and walking by FES in clinical settings $(12,13,15)$. Kagaya and colleagues prepared a muscle stimulation pattern for FES-assisted standing and sitting in paraplegics based on a motion analysis of healthy 


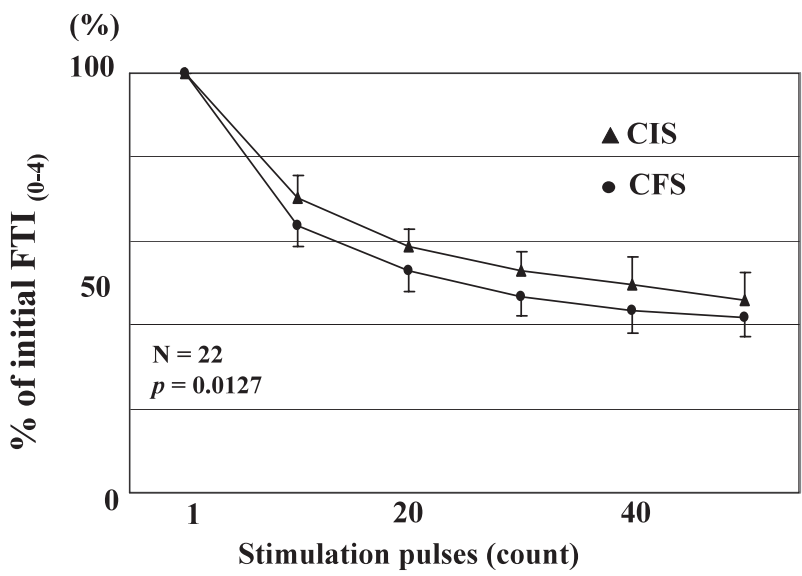

Fig. 6 Time course of \% of initial $\mathrm{FTI}_{(0-4)}$ for CFS and CIS during the muscle fatigue test. Differences in $\mathrm{FTI}_{(0-4)}$ between CFS and CIS were statistically significant (repeated measure ANOVA; $p=0.001)$. Symbols and bars represent mean \pm SD

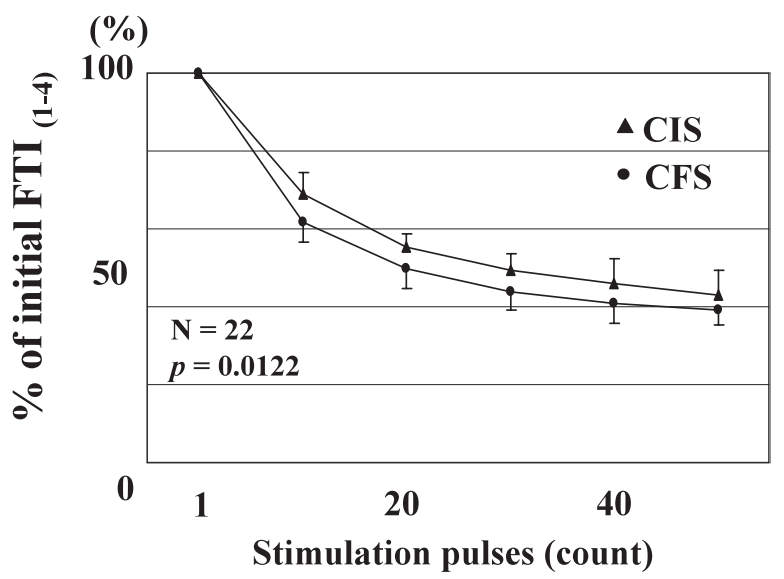

Fig. 8 Time course of \% of initial $\mathrm{FTI}_{(1-4)}$ for CFS and CIS during the muscle fatigue test. Differences in $\mathrm{FTI}_{(1-4)}$ between CFS and CIS were statistically significant (repeated measure ANOVA; $p=0.0122$ ). Symbols and bars represent mean \pm SD

individuals, and reported that it took $3.0 \pm 0.3 \mathrm{sec}$ onds on average for the paraplegics to stand and 3.0 \pm 0.1 seconds to sit (12). To maintain the standing position in paraplegics, Krajl and colleagues applied continuous low-frequency stimulation $(20 \mathrm{~Hz})$ for 1 to 8 seconds with intermittent breaks lasting 1 to 10 seconds and documented the lowest muscle fatigue for 4-second "on"/8-second "off" FES (13). Marsolais and colleagues applied continuous stimulation for 1 to 3 seconds intermittently to the quadriceps femoris, biceps femoris, and tibialis anterior for restoring locomotion in paraplegia by FES (15). In the present study, stimulation for 4 seconds was

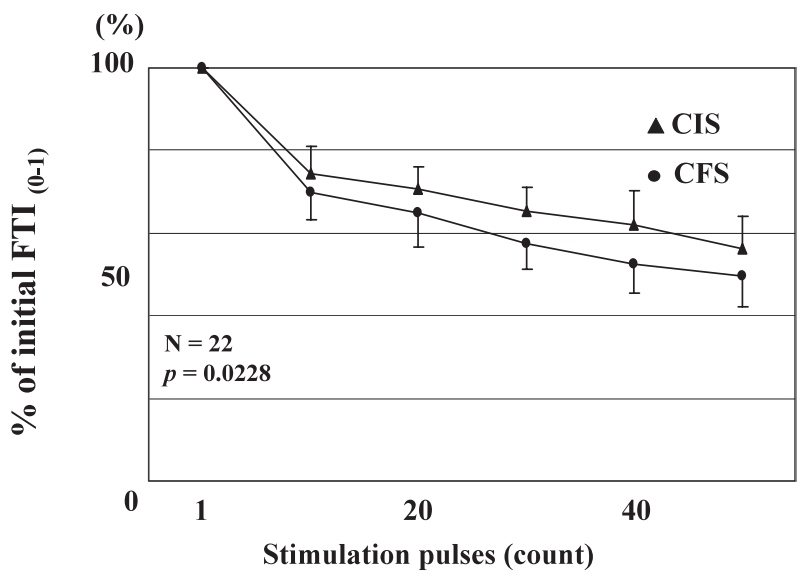

Fig. 7 Time course of \% of initial $\mathrm{FTI}_{(0-1)}$ for CFS and CIS during the muscle fatigue test. Differences in $\mathrm{FTI}_{(0-1)}$ between CFS and CIS were statistically significant (repeated measure ANOVA; $p=0.0228$ ). Symbols and bars represent mean \pm SD

intermittently applied and the condition was similar to FES used in clinical settings. The results of this study should be beneficial for taking advantage of CIS in long-term continuous FES.

There are reports on the physiological mechanisms of the catchlike property. Parmiggiani and colleagues stated that increased muscle stiffness correlated with the catchlike property (18). Duchateau and colleagues reported that the catchlike property occurred when high-frequency stimulation increased $\mathrm{Ca}^{2+}$ release from the sarcoplasmic reticulum (10). In the present study, the difference in muscle fatigue between the CFS and CIS groups was greater in the latter half of the muscle fatigue test. We suppose that the catchlike-inducing effect was more efficiently expressed under low-frequency fatigue because $\mathrm{Ca}^{2+}$ released from sarcoplasmic reticulum is reduced. In this study, chemical analysis was not performed. It will be necessary to investigate the physiological mechanism of the catchlike-inducing effect by use of biochemical tools.

There are some advantages associated with the use of CIS in clinical FES settings. One of the advantages is that muscle force peaks earlier $(5,7)$. In the restoration of standing based on FES incorporating closed-loop control, quick muscular contraction in response to stimulation is needed (16) and as a result, CIS takes advantage of the trains for closed-loop control. Another advantage is the ATP consumption. Ratkevicius and colleagues measured ATP consumption in human quadriceps femoris and clarified that the catchlike property increased the maximum muscle force and workload regardless of 
ATP consumption (20). They applied continuous low-frequency stimulation for $300 \mathrm{msec}$ and the results of our study showed that 4-second continuous stimulation also increased muscular workload. Further studies are necessary to investigate the ATP consumption associated with 4-second continuous stimulation.

When FES incorporating closed-loop control is used for the restoration of lower extremity function, paraplegics can remain standing for 30 to $60 \mathrm{~min}$ utes with the conventional low-frequency stimulation $(9,22)$. The results of the present study showed that the catchlike-inducing low-frequency stimulation takes advantage of the maximum muscle force, workload, and muscle fatigue. CIS may be useful for restoring functions such as standing and walking, and longer restoration may be available by combining CIS with the currently available systems.

\section{REFERENCES}

1. Andrews BJ, Barnett RW, Phillips GF and Kirkwood CA (1989) Rule-based control of a hybrid FES orthosis for assisting paraplegic locomotion. Automedica 11, 175-199.

2. Bevan L, Laouris Y, Reinking RM and Stuart DG (1992) The effects of the stimulation pattern on the fatigue of single motor units in adult cats. $J$ Physiol 449, 85-108.

3. Binder-Macleod SA and Barker CB III (1991) Use of a catchlike property of human skeletal muscle to reduce fatigue. Muscle Nerve 14, 850-857.

4. Binder-Macleod SA and Barrish WJ (1992) Force response of rat soleus muscle to variable-frequency train stimulation. $J$ Neurophysiol 68, 1068-1078.

5. Binder-Macleod SA and Lee SCK (1996) Catchlike property of human muscle during isovelocity movements. $J$ Appl Physiol 80, 2051-2059.

6. Binder-Macleod SA, Lee SCK and Baadte SA (1997) Reduction of the fatigue-induced force decline in human skeletal muscle by optimized stimulation trains. Arch Phys Med Rehabil 78, 1129-1137.

7. Binder-Macleod SA, Lee SCK, Russ DW and Kucharski LJ (1998) Effects of activation pattern on human skeletal muscle fatigue. Muscle Nerve 21, 1145-1152.

8. Burke RE, Rudomin P and Zajac FE III (1970) Catch property in single mammalian motor units. Science 168, 122-124.

9. Davis R, Houdayer T, Andrews B and Barriskill A (1999) Paraplegia: Prolonged standing using closed-loop functional electrical stimulation and Andrews ankle-foot orthosis. Artif Organs 23, 418-420.

10. Duchateau J and Hainaut K (1986) Nonlinear summation of contractions in striated muscle. II. Potentiation of intracellular $\mathrm{Ca}^{2+}$ movements in single barnacle muscle fibres. J Muscle Res Cell Motil 7, 18-24.

11. Hawkins D and Bey M (1994) A comprehensive approach for studying muscle-tendon mechanics. J Biomech Eng 116, $51-55$.

12. Kagaya H, Shimada Y, Ebata K, Sato M, Sato K, Yukawa T and Obinata G (1995) Restoration and analysis of standingup in complete paraplegia utilizing functional electrical stimulation. Arch Phys Med Rehabil 76, 876-881.

13. Krajl A, Bajd T, Turk R and Benko H (1986) Posture switching for prolonging functional electrical stimulation standing in paraplegic patients. Paraplegia 24, 221-230.

14. Liberson WT, Holmquest HJ, Scot D and Dow M (1961) Functional electrotherapy: stimulation of the peroneal nerve synchronized with the swing phase of the gait of hemiplegic patients. Arch Phys Med Rehabil 42, 101-105.

15. Marsolais EB and Kobetic R (1987) Functional electrical stimulation for walking in paraplegia. J Bone Joint Surg 69-A, 728-733.

16. Matsunaga T, Shimada Y and Sato K (1999) Muscle fatigue from intermittent stimulation with low and high frequency electrical pulses. Arch Phys Med Rehabil 80, 48-53.

17. Misawa A, Shimada Y, Matsunaga T and Sato K (2001) The effects of therapeutic electrical stimulation on acute muscle atrophy in rats after spinal cord injury. Arch Phys Med Rehabil 82, 1596-1603.

18. Parmiggiani F and Stein RB (1981) Nonlinear summation of contractions in cat muscles. II. Later facilitation and stiffness changes. J Gen Physiol 78, 295-311.

19. Peckham PH, Keith MW and Freehafer AA (1988) Restoration of functional control by electrical stimulation in the upper extremity of the quadriplegic patient. J Bone Joint Surg 70-A, 144-148.

20. Ratkevicius A and Quistorff B (2002) Metabolic costs of force generation for constant-frequency and catchlike-inducing electrical stimulation in human tibialis anterior muscle. Muscle Nerve 25, 419-426.

21. Shimada Y, Sato K, Abe E, Kagaya H, Ebata K, Oba M and Sato M (1996) Clinical experience of functional electrical stimulation in complete paraplegia. Spinal cord 34, 615-619.

22. Shimada Y, Sato K, Matsunaga T, Tsutsumi Y, Misawa A, Ando S, Minato T, Sato M, Chida S and Hatakeyama K (2001) Closed loop control using a stretch sensor for restration of standing with functional electrical stimulation in complete paraplegia. Tohoku J Exp Med 193, 221-227.

23. Van Lunteren E and Sankey CB (2000) Catchlike property of rat diaphragm: subsequent train frequency effects in variabletrain stimulation. J Appl Physiol 88, 586-598.

24. Zajac FE and Young JL (1980) Properties of stimulus trains producing maximum tension-time area per pulse from single motor units in medial gastrocnemius muscle of the cat. $J$ Neurophysiol 43, 1206-1220. 